\title{
GENE DELETIONS IN JAPANESE PATIENTS WITH DUCHENNE AND BECKER MUSCULAR DYSTROPHY
}

\author{
Jun-ichi Asano, ${ }^{1}$ Shunji Tomatsu, ${ }^{1}$ Kazuko Sukegawa, ${ }^{1}$ Seiji Yamaguchi, ${ }^{1}$ \\ Yuko IKfdo, ${ }^{1}$ Ryoji Minami, ${ }^{2}$ Mitsuo Irda, ${ }^{3}$ Masaaki Nishimura, ${ }^{4}$ \\ Masanori NaKagawa, ${ }^{5}$ Morio OHSHIRo, ${ }^{5}$ and Tadao OrII ${ }^{1}$ \\ ${ }^{1}$ Department of Pediatrics, Gifu University, School of Medicine, Tsukasamachi, Gifu 500, Japan \\ ${ }^{2}$ National Yakumo Hospital, Yakumomachi, Yamakoshi-gun, Hokkaido 049-31, Japan \\ ${ }^{3}$ National Suzuka Hospital, Kasadomachi, Suzuka, Mie 513, Japan \\ ${ }^{4}$ National Nagara Hospital, Nagara, Gifu 502, Japan \\ ${ }^{5}$ National Okinawa Hospital, Ganeko, Ginowan, Okinawa 901-22, Japan
}

\begin{abstract}
Summary Thirty-eight unrelated Japanese patients with Duchenne and Becker muscular dystrophy (DMD and BMD) have been investigated with the DMD cDNA probes. The 14-kb DMD cDNA was subdivided into 6 subclones and HindIII-digested DNAs were analyzed by Southern blotting. Out of 38 unrelated patients, 14 showed a deletion of one or several of the exon-containing HindIII fragments (36.8\%). These corresponded to $50 \%(9 / 18)$ of BMD patients and $25 \%(5 / 20)$ of DMD patients, and the position and extent of deletions were mapped and proved to be more heterogeneous in DMD than in BMD. Both ends of deletions detected in probe $1-2 \mathrm{a}$ were common to all six BMD patients without the maintenance of reading frame of messenger RNA, and $5^{\prime}$ ends of deletions in probe 5b-7 were also common but maintained in frame in three $\mathrm{BMD}$ patients. The phenotypic-specific deletion in Japanese BMD patients has existed in the $5^{\prime}$ end of the DMD gene, although its apparently similar deletion produced a wide range of clinical courses (BMD phenotype). There was no tight correlation between clinical severity and presence or absence of deletion in DMD or BMD.
\end{abstract}

Key Words cDNA, DNA deletion, frame-shift, Southern blotting

\section{INTRODUCTION}

Duchenne muscular dystrophy (DMD) is a severe X-linked recessive disease affecting approximately 1 in 3,500 males. Affected males are generally wheelchairbound by the age of 12 and die in their teens. Becker muscular dystrophy (BMD)

Received November 29, 1989; revised version received December 26, 1989; Accepted December 26,1989 
is a less frequent, milder form with a much longer life span. And it is known BMD is allelic to DMD.

Recently the full-length 14-kb DMD cDNA has been cloned and was completely sequenced by Koenig et al. (1987). The gene product, called dystrophin, was shown to be missing in DMD muscle. On the other hand, dystrophin was shown to be present in BMD muscle (Hoffman et al., 1988). With the cDNA probes, Koenig et al. (1987) detected deletions of HindIII-digested DNAs in more than $50 \%$ of DMD patients. And there have been many reports as for DNA deletions of Caucasian DMD and BMD patients, and no simple correlation between the deletion size or location and the clinical severity of the disease has been observed (Malhotra et al., 1988; Hart et al., 1989; Lindlöf et al., 1989; Baumbach et al., 1989). One possible explanation for phenotypic difference in BMD and DMD has been reported to depend on whether or not the translational reading frame is maintained and some functional protein is produced (Monaco et al., 1988; Forrest et al., 1988), and this theory was proved to be true of $92 \%$ of Caucasian DMD and BMD patients (Koenig et al., 1989). But in some BMD patients, the deletions disrupted the translational reading frame, indicating that other factors, which compensate for the frame-shift mutation should exist (Malhotra et al., 1988; Baumbach et al., 1989).

Sugino et al. (1989) reported that DNA deletions were detected in $43 \%$ of Japanese DMD patients, and deletion hot spots were also located in the proximal region and the central region of the gene.

To obtain further information on intragenic deletions in Japanese DMD and BMD patients, and to gain an understanding of the relationship between deletions and clinical features, we have studied 38 unrelated DMD and BMD patients by using Southern blot analysis. We discuss here frame shift deletions in patients with DMD and BMD.

\section{SUBJECTS AND METHODS}

Subjects. Blood was obtained from 38 unrelated Japanese BMD and DMD males. The probands were diagnosed prior to DNA analysis by using standard criteria for BMD and/or DMD in conjunction with highly elevated serum creatine kinase (CK) levels and typical dystrophic myopathic changes on muscle biopsy. These patients were classified as DMD or BMD according to their clinical features; (1) DMD (patients wheelchair-bound before the age of 12 years), (2) BMD (patients wheelchair-bound at a later age or not at all; wheelchair-bound patients surviving after the age of 30 even if they first became wheelchair-bound before the age of 12). Patients too young to be classified were excluded. The patients consisted of 20 DMD individuals and 18 BMD individuals. Of the 38 patients, 4 were regarded as having mental retardation, as their IQ's were below 70 .

Methods. The 14-kb DMD cDNA (Koenig et al., 1987) received from the American Type Culture Collection was subdivided and was made available as 6 
subclones (1-2a, 2b-3, 4-5a, 5b-7, 8, 9-14).

About $5 \mu \mathrm{g}$ of genomic DNA was digested with restriction enzyme HindIII (Takara Shuzo, Japan), electrophoresed in $0.6 \%$ agarose gels and blotted onto Nylon filters (Hybond N; Amersham, U.K.) according to the methods of Southern (1975). Following UV transillumination for $5 \mathrm{~min}$, the filters were hybridized with the above six probes labeled by the multiprime labeling system and $\left[\alpha^{-32} \mathrm{P}\right] \mathrm{dCTP}$ $(3,000 \mathrm{Ci} / \mathrm{mmol})$ from Amersham. The filters were washed in $2 \times \mathrm{SSC}, 0.1 \% \mathrm{SDS}$ for 20 min twice at room temperature and then in $0.1 \times \mathrm{SSC}, 0.1 \% \mathrm{SDS}$ for $20 \mathrm{~min}$ twice at $65^{\circ} \mathrm{C}$. Autoradiography was carried out at $-80^{\circ} \mathrm{C}$ on Kodak X-ray film with two intensifying screens for 3-7 days. A list of the probes as well as the proposed order of detectable exon-containing fragments produced after digestion with HindIII is presented in Table 1.

\section{RESULTS}

The 38 patients (18 BMD and 20 DMD individuals) were examined for intragenic deletions using all the cDNA probes. Fourteen unrelated patients showed deletions with at least one probe, and the deletion frequency was $36.8 \%$. Nine of the 18 BMD patients $(50 \%)$ and 5 of the 20 DMD patients $(25 \%)$ had a deletion. The extent and location of the deletions relative to the exon-containing HindIII fragments and cDNA probes are schematically summarized in Table 1.

The deletions detected by using the cDNAs existed unevenly throughout the DMD gene, and all deletions belonged to either of two deletion hot spots in the proximal region or the central region of the gene. No deletions were detected except probes 1-2a, 5b-7, and 8. The breakpoints of these deletions in DMD patients were distributed more heterogeneously than in BMD patients.

In BMD, all six patients who had deletions with probe 1-2a had both of the common ends of deleted exons and three other patients had the common $5^{\prime}$ end with probe 5b-7. On the other hand, in DMD, both ends of deletions in probe 5b-7 and 8 were different. Only one DMD patient had a junctional fragment with probe 8. Results of probe 1-2a on 9 BMD and 6 DMD patients are shown in Fig. 1, and those of probe 8 on 7 BMD and 6 DMD patients are shown in Fig. 2.

Correlations between clinical features and presence or absence of deletion are shown in Tables 2 and 3 for DMD and BMD patients, respectively. As for family history, period of walking unaided, age in wheelchair, mental retardation and CK on admission, there was no clear discrimination between DMD patients with and without deletions. BMD patients with deletions became wheelchair-bound earlier, and their levels of CK tended to be higher, although the number of cases was not sufficient to allow us to conclude that this is generally the case. Other clinical manifestations had no significant difference between BMD patients with and without deletions.

Six BMD patients displayed apparently identical deletions in probe 1-2a, but 


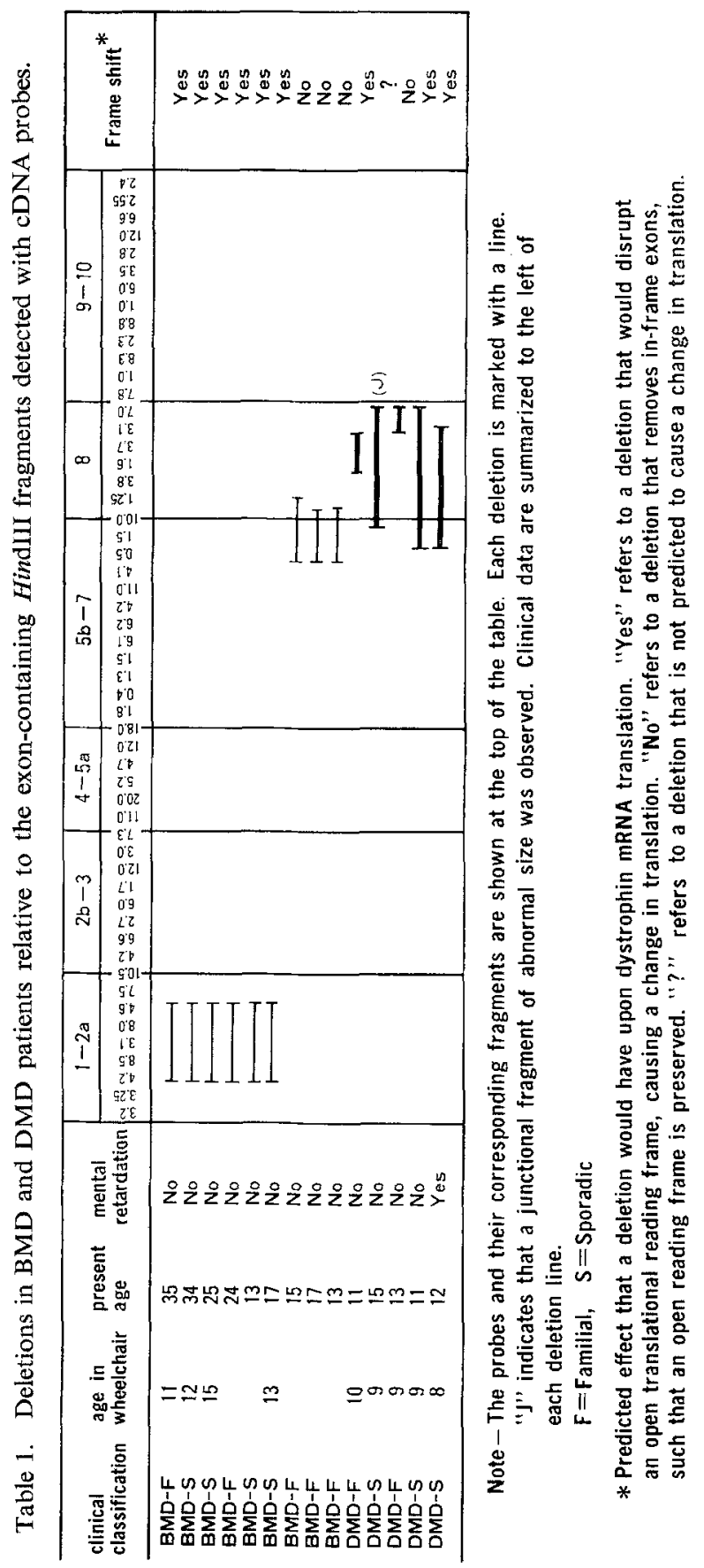




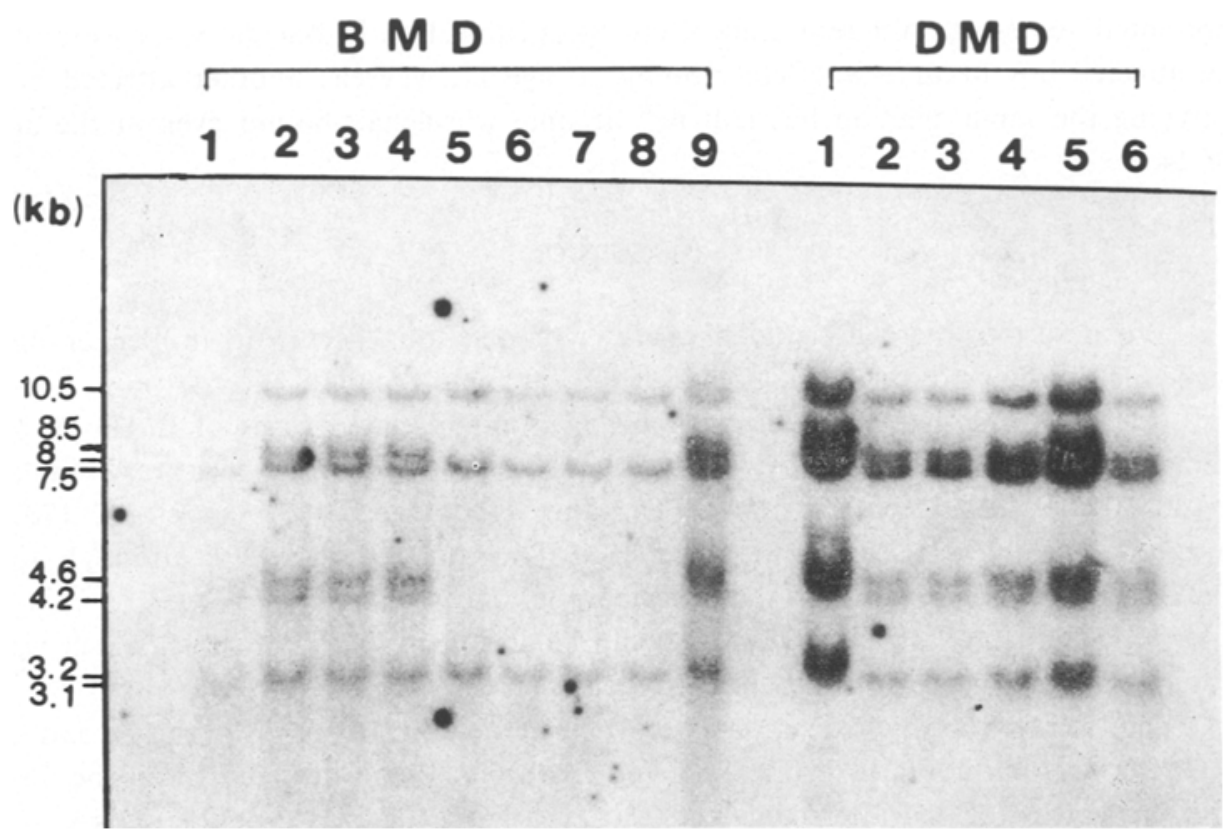

Fig. 1. Autoradiography of probe 1-2a on $9 \mathrm{BMD}$ patients and $6 \mathrm{DMD}$ patients. Common deletions of 8.5, 8.0, 4.6, 4.2, $3.1 \mathrm{~kb}$ were detected in BMD 5, 6, 7, 8, but no deletions were detected in DMD.

(a)

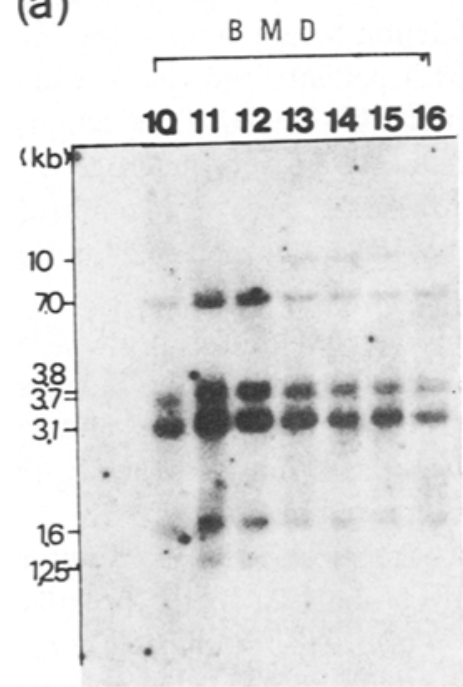

(b)
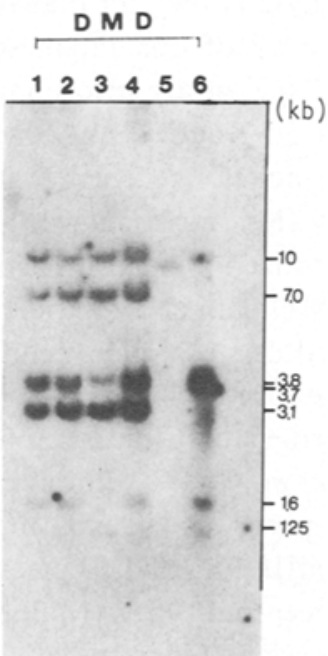

Fig. 2. Autoradiography of probe 8 on 7 BMD patients and 6 DMD patients. (a) In BMD $10,10 \mathrm{~kb}$ and $1.25 \mathrm{~kb}$ were deleted. In BMD 11 and $12,10 \mathrm{~kb}$ was commonly deleted. (b) In DMD 3, 3.7 kb and $1.6 \mathrm{~kb}$ were deleted. In DMD 5, all fragments were deleted and junctional fragment $(\mathrm{J})$ was detected. In DMD $6,7.0 \mathrm{~kb}$ and $3.1 \mathrm{~kb}$ were deleted. 
presented somewhat different clinical courses (Table 1). In one case, for example, an affected boy became wheelchair-bound at age 11, whereas another affected boy carrying the same deletion has still not become wheelchair-bound even at the age of 24 .

\section{DISCUSSION}

We described here a detailed analysis of deletions occurring in Becker and Duchenne muscular dystrophy patients of the Japanese population.

We have identified gene deletions in $36.8 \%$ of unrelated cases of BMD $(50 \%)$ and DMD (25\%), using cDNAs for the DMD gene. That value was very similar to $30-60 \%$ values recently reported by other laboratories (Koenig et al., 1987; Burghes et al., 1987; Forrest et al., 1987, 1988; Hart et al., 1989), although the value in $\mathrm{DMD}$ was lower than the expected one.

All deletions of BMD and DMD could be detected in two hot spots, which was in agreement with previously published results (Koenig et al., 1987).

One region in which deletions were detected frequently is near the $5^{\prime}$ end of the gene corresponding to probe $1-2 \mathrm{a}$ and the other region detected by probe $5 \mathrm{~b}-7$ and probe 8 is located approximately 6 to $7 \mathrm{~kb}$ from the $5^{\prime}$ end of the cDNA and is near the middle of the gene.

No deletions other than these three probes were detected, and there was no identical deletion pattern between BMD and DMD.

Although deletion spots are unevenly distributed throughout the DMD gene, even more striking is the fact that the deletion breakpoints in the probe 1-2a region are common to all of our Japanese BMD patients and the $5^{\prime}$ end breakpoints in the probe $5 \mathrm{~b}-7$ region are also common to all. In DMD, deletions were detected only in probes $5 \mathrm{~b}-7$ and 8 , and the deletions were very heterogeneous.

The same deletions detected in probe 1-2a were also reported in Caucasian BMD patients (Koenig et al., 1989), but deletions detected in Caucasian BMD patients are localized in several parts of probe 1-2a.

Our six BMD patients with the common breakpoints in probe 1-2a are unrelated, so this identified deletion might tend to occur especially in the Japanese BMD group. In contrast, the same deletion pattern detected by probes $5 \mathrm{~b}-7$ and 8 in our patients has been described in Caucasian BMD patients most frequently (Norman et al., 1989; Lindlöf ct al., 1989), although not reported in DMD.

Since the positions of exon-intron borders and the distribution of the exons within the HindIII fragments detected by probe $1-2$ a to the proximal region of 10 have recently been published (Malhorta et al., 1988; Baumbach et al., 1989; Koenig et al., 1989), we were able to investigate the hypothesis that the phenotypic differences between BMD and DMD depend upon whether or not the translational reading frame is maintained. The deletions of 4.2 to $4.6 \mathrm{HindIII}$ fragments in the probe 1-2a lead to apparent frame shift (Tables 1-4). This disruption of the open reading frame occurs at the extreme $5^{\prime}$ end of the gene. The resulting BMD phenotype 
Table 2. Relationship between cDNA deletion and clinical features in DMD patients.

\begin{tabular}{lcc}
\hline & Deletion $(+)$ & Deletion $(-)$ \\
\hline Familial case & $2 / 5$ & $4 / 15$ \\
Walk unaided (months) & $15.4 \pm 3.1$ & $15.9 \pm 2.6$ \\
Age in wheelchair (years) & $9.8 \pm 1.7$ & $10.5 \pm 1.4$ \\
Mental retardation & $1 / 5$ & $3 / 15$ \\
CK $(\mathrm{IU} / \mathrm{L})$ & $4,193 \pm 4.195$ & $2,243 \pm 2,271$ \\
\hline
\end{tabular}

Table 3. Relationship between cDNA deletion and clinical features in BMD patients.

\begin{tabular}{lcc}
\hline & Deletion $(+)$ & Deletion $(-)$ \\
\hline Familial case & $5 / 9$ & $3 / 9$ \\
Walk unaided (months) & $14.3 \pm 1.9$ & $13.5 \pm 1.7$ \\
Age in wheelchair (years) & $12.8 \pm 1.5$ & $16.8 \pm 3.5$ \\
& $(\mathrm{n}=4)$ & $(\mathrm{n}=7)$ \\
CK (IU/L) & $2,449 \pm 2,115$ & $643 \pm 456$ \\
\hline
\end{tabular}

No cases had mental retardation.

could be explained by the additional change that might maintain the translational reading frame. On the other hand, the deletions detected by probes $5 b-7$ and 8 would not disrupt the mRNA reading frame, and most of the previous BMD reports of deletions in this central region also support the maintenance of the reading frame.

The potential mechanism to compensate for the frame shift has been postulated as follows: in the $5^{\prime}$ end of the DMD gene, translational reinitiation produces the partial functional dystrophin molecule or alternative mRNA splicing mechanism such as exon skipping and cryptic site utilization, and the presence of a secondary promoter downstream from the deletion can correct for a frame shift. As for DMD patients, it was predicted that three out of four patients would produce frame shift.

Interestingly, BMD patients bearing apparently identical deletions within the $5^{\prime}$ end of the gene gave somewhat different clinical courses (Table 1). This suggested that in addition to the deletion pattern, secondary factors such as individual genetic backgrounds should play an important role in deciding the disease phenotype.

In both BMD and DMD cases, we have found no difference in clinical severity between cases with deletions and without deletions.

Our study is the first to reveal the phenotypic difference of the deletion pattern between Japanese BMD and DMD, and especially in BMD patients, the common deletion around the $5^{\prime}$ end of the gene has been observed. 


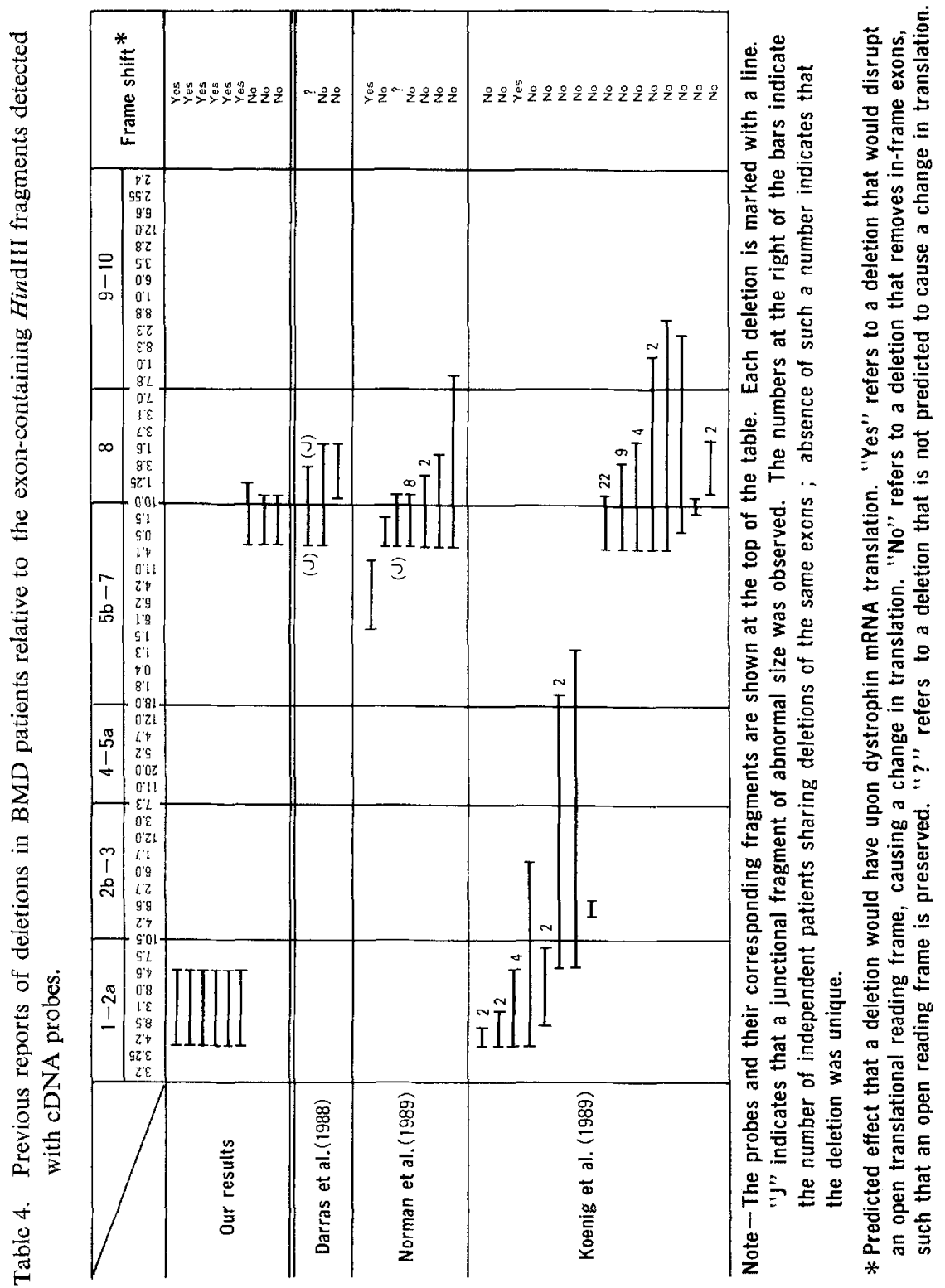


Acknowledgments Our study was supported in part by a grant for the promotion of science research (No. 61440044) from the Ministry of Education, Science and Culture of Japan.

\section{REFERENCES}

Baumbach, L.L., Chamberlain, J.S., Ward, P.A., Farwell, N.J. and Caskey, C.T. 1989. Molecular and clinical correlations of deletions leading to Duchenne and Becker muscular dystrophies. Neurology 39: 465-474.

Burghes, A.H.M., Logan, C., Hu, H., Belfall, B., Worton, R. and Ray, P.N. 1987. Isolation of a cDNA clone from the region of an X;21 translocation that breaks within the Duchenne/Becker muscular dystrophies. Nature 328: 434-437.

Darras, B.T., Blattner, P., Harper, J.F., Spiro, A.J., Alter, S. and Francke, U. 1988. Intragenic deletions in 21 Duchenne muscular dystrophy (DMD)/Becker muscular dystrophy (BMD) families studied with the dystrophin cDNA: location of breakpoints on HindIII and BglII exoncontaining fragment maps, mitotic origin of the mutations. Am. J. Hum. Genet. 43: 620-629.

Forrest, S.M., Cross, G.S., Speer, A., Gardner-Medwin, D., Burnas, J. and Davies, K.E. 1987. Preferential deletion of exons in Duchenne and Becker muscular dystrophies. Nature 329: 638-640.

Forrest, S.M., Cross, G.S., Flint, T., Speer, A., Robson, K.J.H. and Davies, K.E. 1988. Further studies of gene deletions that cause Duchenne and Becker muscular dystrophies. Genomics 2: 109-144.

Hart, K.A., Abbs, S., Wapenaar, M.C., Cole, C.G., Hodgson, S.V. and Bobrow, M. 1989. Molecular deletions in the Duchenne/Becker muscular dystrophy gene. Clin. Genet. 35: 251-260.

Hoffman, E.P., Fishbeck, K.H., Brown, R.H., Johnson, M., Medori, R., Loike, J.D., Harris, J.B., Waterson, R., Brooke, M., Specht, L., Kupsky, W., Chamberlain, J., Caskey, C.T., Shapiro, F. and Kunkel, L.M. 1988. Characterization of dystrophin in muscle-biopsy specimens from patients with Duchenne's and Becker's muscular dystrophy. N. Engl. J. Med. 318: 13631368.

Koenig, M., Hoffmann, E.P., Bertelson, C.J., Monaco, A.P., Feener, C. and Kunkel, L.M. 1987. Complete cloning of the Duchenne muscular dystrophy (DMD) cDNA and preliminary genomic organization of the DMD gene in normal and affected individuals. Cell 50: 509-517.

Koenig, M., Beggs, A.H., Moyer, M., Scherph, S., Heindrich, K., Bettecken, T., Meng, G., Muller, C.R., Lindröf, M., Kaariainen, H., de la Chapelle, A., Kiuru, A., Savontaus, M.L., Gilgenkrantz, H., Recan, D., Chelly, J., Kaplan, J.C., Covone, A.E., Archidiacono, N., Romeo, G., Liechti-Gallati, S., Schneider, V., Braga, S., Moser, H., Darras, B.T., Murphy, P., Francke, U., Chen, J.D., Morgan, G., Denton, M., Greenberg, C.R., van Ommen, G.J.R. and Kunkel, L.M. 1989. The molecular basis for Duchenne versus Becker muscular dystrophy: correlation of severity with type of deletion. Am. J. Hum. Genet. 45: 498-506.

Lindlöf, M., Kiuru, A., Kaarainen, H., Kalimo, H., Lang, H., Pihko, H., Ropola, J., Somer, H., Somer, M., Savontaus, M.L. and Chapelle, A. 1989. Gene deletions in X-linked muscular dystrophy. Am. J. Hum. Genet. 44: 496-503.

Malhotra, S.B., Hart, K.A., Klamut, H.J., Thomas, N.S.T., Bodrug, S.E., Burghes, A.H.M., Bobrow, M., Harper, P.S., Thompson, M.W., Ray, P.N. and Worton, R.G. 1988. Frame-shift deletions in patients with Duchenne and Becker muscular dystrophy. Science 242: 755-759.

Medori, R., Brooke, M.H. and Waterston, R.H. 1989. Genetic abnormalities in Duchenne and Becker dystrophies: clinical corelations. Neurology 39: 461-465.

Monaco, A.P., Bertelson, C.J., Liechti-Gallati, S., Moser, H. and Kunkel, L.M. 1988. An explanation for the phenotypic differences betwcen patients bearing partial deletions of the DMD locus. Genomics 2: 90-95.

Norman, A., Thomas, N., Coakley, J. and Harper, P. 1989. Distinction of Becker from limb-girdle 
muscular dystrophy by means of dystrophin cDNA probes. Lancet 4: 466-468.

Southern, E.M. 1975. Detection of specific sequences among DNA fragments separated by gel electrophoresis. J. Mol. Biol. 48: 503-517.

Sugino, S., Fujishita, S., Kamimura, N., Matsumoto, T., Wapenaar, M.C., Deng, H.X., Shibuya, M., Miike, T. and Niikawa, N. 1989. Molecular-genetic study of Duchenne and Becker muscular dystrophies: deletion analyses of 45 Japanese patients and segregation analyses in their families with RFLPs based on the data from normal Japanese females. Am. J. Med. Genet. 34, 555-561. 